Comportamento de Podisus nigrispinus (Dallas) (Hemiptera: Pentatomidae) na interação com lagartas de Spodoptera cosmioides (Walker) (Lepidoptera: Noctuidae) e cultivares de soja com diferentes graus de resistência

\title{
COMPORTAMENTO DE Podisus nigrispinus (Dallas) (HEMIPTERA: PENTATOMIDAE) NA INTERAÇÃO COM LAGARTAS DE Spodoptera cosmioides (Walker) (LEPIDOPTERA: NOCTUIDAE) E CULTIVARES DE SOJA COM DIFERENTES GRAUS DE RESISTENNCIA
}

\author{
Daline Benites Bottega ${ }^{1}$; Bruno Henrique Sardinha de Souza ${ }^{1}$; Anderson Gonçalves da Silva ${ }^{1}$; \\ Arlindo Leal Boiça Júnior ${ }^{1}$
}

${ }^{1}$ Faculdade de Ciências Agrárias e Veterinárias - UNESP, Departamento de Fitossanidade, Laboratório de Resistência de Plantas a Insetos, E-mail: daline4@bol.com.br; souzabhs@gmail.com; agroanderson.silva@yahoo.com.br; aboicajr@fcav.unesp.br

\section{RESUMO}

Objetivou-se com o presente trabalho avaliar o comportamento de atratividade e predação de Podisus nigrispinus (Dallas) em relação a lagartas de Spodoptera cosmioides (Walker) alimentadas com folhas de cultivares de soja. Foram avaliadas a atratividade e a predação de $P$. nigrispinus em resposta a odores emitidos por $S$. cosmioides, por folhas de plantas de soja (cultivar BR 16) e por fezes do herbívoro (alimentado com a mesma cultivar); e a interação entre resistência de plantas e controle biológico, por meio da avaliação da atratividade e da predação de $P$. nigrispinus sobre lagartas de $S$. cosmioides alimentadas com cultivares de soja com diferentes graus de resistência a insetos, quais sejam, IAC 100 (resistente), Dowling (resistente), BR 16 (suscetível) e IGRA RA 626 RR (suscetível). Concluiu-se que os voláteis liberados pela lagarta de $S$. cosmioides estimulam o predador a encontrar a presa. As cultivares resistentes de soja IAC $100 \mathrm{e}$ Dowling, utilizadas para alimentação das lagartas de $S$. cosmioides, não afetam a atratividade e a predação de $P$. nigrispinus. A associação entre as cultivares resistentes e o controle biológico com $P$. nigrispinus pode apresentar efeito sinérgico no controle de $S$. cosmioides em campo, com potencial para ser incluído em um programa de MIP para a cultura da soja.

Palavras-chave: resistência extrínseca, predador, interação tritrófica, voláteis, MIP

\section{BEHAVIOR OF Podisus nigrispinus (Dallas) (HEMIPTERA: PENTATOMIDAE) IN THE INTERACTION WITH Spodoptera cosmioides (Walker) (LEPIDOPTERA: NOCTUIDAE) LARVAE AND SOYBEAN CULTIVARS WITH DIFFERENT DEGREES OF RESISTANCE}

\section{ABSTRACT}

The aim of this study was to evaluate the attractiveness and predation behavior of Podisus nigrispinus (Dallas) towards Spodoptera cosmioides (Walker) fed on leaves of soybean cultivars. Attractiveness and predation of $P$. nigrispinus were recorded in response to odors emitted by $S$. cosmioides, leaves of soybean (cultivar BR 16), and feces of the herbivore (fed on the same cultivar); the interaction of plant resistance and biological control was also evaluated through the attractiveness and predation of $P$. nigrispinus on larvae of $S$. cosmioides fed soybean cultivars with different degrees of resistance against insects, IAC 100 (resistant), Dowling (resistant), BR 16 (susceptible), and IGRA RA 626 RR (susceptible). We concluded the volatiles released by $S$. cosmioides larvae stimulate the predator to find the prey. The resistant cultivars of soybean IAC 100 and Dowling, used to feed the larvae of $S$. cosmioides, do not affect attractiveness and predation by $P$. nigrispinus. Association between resistant plants and biological control using $P$. nigrispinus may produce a synergic effect on the control of $S$. cosmioides in the field, and can be potentially included in an IPM program for soybean.

Keywords: extrinsic resistance, predator, tritrophic interaction, volatiles, IPM 


\section{INTRODUÇÃO}

Diversos estudos têm examinado as possibilidades de integração entre diferentes táticas de controle de pragas, como por exemplo, as interações tróficas entre plantas, pragas e seus inimigos naturais, sejam parasitoides (KAROWE \& SCHOONHOVEN, 1992) ou predadores (ISENHOUR et al., 1989), de modo que o conhecimento do comportamento do inimigo natural nessas interações é de extrema importância para maximizar sua ação no contexto do manejo integrado de pragas (MIP).

No Brasil, o percevejo predador Podisus nigrispinus (Dallas, 1851) (Hemiptera: Pentatomidae) tem sido encontrado predando diferentes pragas em diversas culturas (TORRES et al. 1996; DE CLERCQ, 2000). Esse inseto apresenta elevada capacidade predatória de lagartas da traça-do-tomateiro Tuta absoluta (Meyrick, 1917) (Lepidoptera: Gelechiidae), em laboratório, e Chrysodeixis chalcites (Esper, 1789) (Lepidoptera: Noctuidae) e Spodoptera spp. (Lepidoptera: Noctuidae) em cultivos protegidos (DE CLERCQ, 2000), sendo considerado um potencial agente de controle biológico para o manejo de lagartas desfolhadoras.

Vários estudos com plantas resistentes e sua influência sobre inimigos naturais (resistência extrínseca) vem sendo realizados, assim como o estudo do comportamento do inimigo natural na localização da presa devido aos voláteis emitidos quer seja pelas plantas, excrementos ou pelo herbívoro (RICE \& WILDE, 1989; PFANNESTIEL et al., 1995; BOIÇA JÚNIOR et al., 2002). Percevejos predadores, em geral, localizam suas presas por meio de fezes, sinais visuais a pequenas distâncias ou por estímulos vibratórios, emitidos durante o processo de alimentação da presa na superfície foliar (PFANNESTIEL et al., 1995).
Esses estímulos geralmente são decorrentes de substâncias do metabolismo secundário das plantas que, na maioria das vezes, relacionam-se à defesa (PRICE, 1984), e podem estar envolvidas na interação entre as plantas e o segundo ou terceiro níveis tróficos, atuando como alomônios, cairomônios ou sinomônios, dependendo do contexto ecológico (SEIGLER \& PRICE, 1976). Esses voláteis emitidos pelas plantas, além de atuarem como mecanismo de defesa, podem agir diretamente exercendo um efeito negativo sobre os herbívoros (VET \& DICKE, 1992) e/ou atuar indiretamente, atraindo seus inimigos naturais, sendo que essa atração pode causar benefícios (RUBERSON et al., 1986), malefícios ou não terem efeito (VIVAN et al., 2003) sobre eles. Como exemplo de estudos dessas interações, Cavalcanti et al. (2000) citam que o predador $P$. nigrispinus pode localizar lagartas de Thyrinteina arnobia (Stoll, 1782) (Lepidoptera: Geometridae), praga desfolhadora de eucaliptos, combinando sinomônios da planta hospedeira e cairomônios das fezes e do corpo da presa. Larvas de Hippodamia convergens (GuerinMeneville, 1842) (Coleoptera: Coccinellidae) alimentadas com o pulgão Schizaphis graminum (Rondani, 1852) (Hemiptera: Aphididae) criado em cultivares de sorgo com resistência do tipo antibiose, apresentaram menor viabilidade larval e pupal e maior período de desenvolvimento (RICE \& WILDE, 1989). Boiça Júnior et al. (2002) constataram maior consumo de $P$. nigrispinus por lagartas de Alabama argillacea (Hübner, 1818) (Lepidoptera: Noctuidae) criadas em folhas de algodoeiro dos genótipos com maior grau de resistência, indicando compatibilidade entre a utilização de plantas resistentes e o controle biológico.

Para o presente estudo, utilizaram-se lagartas de Spodoptera cosmioides (Walker, 1858) (Lepidoptera: Noctuidae), espécie altamente polífaga, cujos surtos estão relacionados, geralmente, a desequilíbrios 
Comportamento de Podisus nigrispinus (Dallas) (Hemiptera: Pentatomidae) na interação com lagartas de Spodoptera cosmioides (Walker) (Lepidoptera: Noctuidae) e cultivares de soja com diferentes graus de resistência

provocados pelo uso excessivo de inseticidas de amplo espectro (HABIB et al., 1983).

Com a possibilidade de associar a resistência de plantas e o controle biológico, há necessidade de estudos mais profundos sobre as inter-relações existentes no complexo planta-praga-inimigo natural.

Assim, é necessária a integração entre os métodos de controle e os estudos de comportamento para o entendimento das interações que ocorrem entre os organismos em uma cadeia alimentar, para consequentemente se obter melhor eficiência desses métodos em um programa de manejo integrado de pragas (MIP). Desse modo, o objetivo do presente trabalho foi avaliar o comportamento de $P$. nigrispinus na interação com lagartas de $S$. cosmioides alimentadas com diferentes cultivares de soja.

\section{MATERIAL E MÉTODOS}

O experimento foi conduzido em laboratório, à temperatura de $25 \pm 1^{\circ} \mathrm{C}$, UR de $70 \pm 10 \%$ e fotofase de $12 \mathrm{~h}$.

Os percevejos predadores utilizados nos experimentos foram provenientes da criação estoque mantida em laboratório, de acordo com a metodologia de Zanuncio et al. (2001), e as lagartas de $S$. cosmioides foram criadas com dieta artificial, à base de feijão, germe de trigo, proteína de soja, caseína e levedura de cerveja (Greene et al., 1976). As folhas das cultivares de soja foram retiradas de plantas com aproximadamente 60 dias de idade mantidas em vasos de $5 \mathrm{~L}$ de volume contendo terra, areia e esterco à proporção de 1:1:1 e acondicionadas em casa de vegetação.

Para estudar o comportamento de atratividade e predação de ninfas e adultos de $P$. nigrispinus sobre lagartas de $S$. cosmioides foi utilizado um olfatômetro constituído de quatro compartimentos e um fluxo de ar para possibilitar a difusão dos voláteis, a fim de detectar odores provenientes dos diferentes tratamentos. Para a confecção do olfatômetro foram utilizadas arenas circulares de PVC
(8,0 cm de diâmetro) vedadas nas partes superiores e inferiores com tampa de acrílico, sendo que na tampa superior havia um orifício para a saída do fluxo de ar. A sucção do ar foi produzida por um aparelho de inalação (Nevoni 6006), ligado por meio de uma mangueira de silicone até as arenas. Os compartimentos onde foram acondicionados os diferentes tratamentos eram localizados na parede da arena e constituídos de mangueira de silicone (2,54 cm de diâmetro).

Para o teste de atratividade de $P$. nigrispinus a odores emitidos por $S$. cosmioides, alimentada da cv. BR16, folhas de plantas de soja (cv. BR 16) e fezes do herbívoro, os ensaios foram realizados utilizando-se o delineamento experimental de blocos casualizados com quatro tratamentos, lagarta; lagarta + folha; lagarta + fezes; lagarta + folha + fezes, a fim de se estabelecer qual tratamento é o mais atrativo ao predador. Lagartas de quarto ínstar de $S$. cosmioides foram acondicionadas entre pedaços de tecidos voile dentro da mangueira de silicone $(2,54 \mathrm{~cm}$ de diâmetro) a fim de se evitar sua fuga, colocando-se atrás do voile externo uma folha sem qualquer injúria e fezes do referido tratamento, de modo que as lagartas e os percevejos não tinham contato com as folhas ou fezes. Os testes foram realizados com ninfas de terceiro ínstar (oito por repetição) e adultos (quatro por repetição), em testes independentes, avaliando-se o número de $P$. nigrispinus atraídos e número de $P$. nigrispinus predando lagartas de $S$. cosmioides a 1, 3, 5, 10, 30, 60, 120 e 180 minutos após a liberação dos insetos.

Para o teste de atratividade de $P$. nigrispinus por lagartas de $S$. cosmioides alimentadas em cultivares de soja, utilizou-se em cada compartimento do olfatômetro uma lagarta de quarto ínstar de S. cosmioides alimentada com folhas de cultivares de soja com diferentes graus de resistência a insetos, quais sejam IAC 100 resistente a Anticarsia gemmatalis (OLIVEIRA et al., 1993; 
LOURENÇÃO et al., 2000) e Spodoptera eridania (SOUZA et al., 2012), Dowling resistente a Aphis glycines (HILL et al., 2004; LI et al., 2004; CHIOZZA et al., 2010) BR 16 (suscetível) (PIUBELLI et al., 2003; 2005) e IGRA RA 626 RR (suscetível) (SOUZA, 2011).

As lagartas utilizadas nesse experimento foram retiradas previamente da criação de manutenção logo após atingirem o $3^{\text {o }}$ ínstar e transferidas para recipientes plásticos de $15 \mathrm{~cm}$ de diâmetro e $10 \mathrm{~cm}$ de altura, cobertos na parte superior com tecido voile, onde foram alimentadas por quatro dias com folhas das respectivas cultivares de soja. As fezes empregadas no teste foram retiradas desse recipiente, oriundas da alimentação também das folhas de cada cultivar.

Após quatro dias, as lagartas, já no $4^{\circ}$ ínstar, foram acondicionadas entre pedaços de tecidos voile dentro da mangueira de silicone $(2,54 \mathrm{~cm}$ de diâmetro), a fim de se evitar sua fuga, colocando-se atrás do voile externo uma folha sem qualquer injúria e fezes do referido tratamento, de modo que as lagartas não tinham contato com as folhas ou fezes.

Os testes foram realizados com ninfas e adultos do predador, utilizando-se 10 repetições, onde foram liberadas oito ninfas de terceiro ínstar ou quatro adultos, por repetição, avaliando-se o número de $P$. nigrispinus atraídos e número de percevejos que estavam predando as lagartas após 1, 3, 5, $10,15,30,60,120$ e 180 minutos.

Os dados foram submetidos à análise de variância (ANOVA) pelo teste F, e quando significativo, as médias foram comparadas pelo teste de Tukey, ao nível de $5 \%$ de probabilidade.

Realizou-se ainda análise de regressão a fim de se correlacionar a resposta de atratividade de ninfas ou adultos do predador aos tratamentos em função dos diferentes tempos.

\section{RESULTADOS E DISCUSSÃO}

No teste de atratividade de $P$. nigrispinus a odores emitidos por $S$. cosmioides, por folhas de plantas de soja (cultivar BR 16) e por fezes do herbívoro, não houve diferença significativa para o número médio de ninfas ou de adultos do predador nos diferentes tratamentos (Tabelas 1 e 2). No entanto, houve tendência de maior atratividade pelas ninfas no tratamento constituído por lagarta + fezes a um minuto de avaliação e essa se manteve até o final do experimento aos 180 minutos (Tabela 1).

Para o parâmetro predação de lagartas de $S$. cosmioides por ninfas do percevejo, verificou-se atratividade inicial bastante reduzida, atingindo, após 180 minutos, médias 1,$00 ; 1,10$ e 1,00 para os tratamentos lagarta + folha, lagarta + fezes e lagarta + folhas + fezes, respectivamente. Nesse mesmo tempo, no tratamento apenas com lagartas havia em média 0,30 ninfas do percevejo em atividade de predação (Tabela 1). Na avaliação da atratividade de adultos de $P$. nigrispinus, observou-se que para todos os tratamentos e tempos de avaliação houve uma resposta muito parecida, sem diferença estatística entre os tratamentos (Tabela 2).

Já para predação, observou-se um comportamento semelhante aos das ninfas, em que no tratamento constituído apenas por lagartas houve tendência de menor predação $(0,20)$, em comparação aos tratamentos lagarta + folha, lagarta + fezes e lagarta + folha + fezes $(0,60 ; 0,60$ e 0,70 , respectivamente). Esses resultados demonstram que os odores envolvidos favorecem, de forma positiva ou negativa, o reconhecimento da praga-alvo pelo predador e que os voláteis emitidos pela lagarta são atraentes ao predador, mas que um conjunto desses voláteis pode influenciar e atuar de forma sinérgica na localização de $S$. cosmioides pelo predador. 
Comportamento de Podisus nigrispinus (Dallas) (Hemiptera: Pentatomidae) na interação com lagartas de Spodoptera cosmioides (Walker) (Lepidoptera: Noctuidae) e cultivares de soja com diferentes graus de resistência

Tabela 1. Número médio de ninfas de $3^{\circ}$ ínstar de Podisus nigrispinus, atraídos e predando lagartas de Spodoptera cosmioides nos diferentes tratamentos e tempos de avaliação. Temperatura: $25 \pm 1{ }^{\circ} \mathrm{C}$; U.R..: $70 \pm 10 \%$; Fotofase: 12 horas.

\begin{tabular}{|c|c|c|c|c|c|c|c|c|c|}
\hline \multirow[b]{3}{*}{ TRATAMENTOS } & \multicolumn{9}{|c|}{ Ninfas de $P$. nigrispinus atraídas por lagartas de $S$.cosmioides } \\
\hline & \multicolumn{9}{|c|}{\begin{tabular}{|c|} 
TEMPOS $^{1}$ \\
\end{tabular}} \\
\hline & $1^{\prime}$ & $3^{\prime}$ & $5^{\prime}$ & $10^{\prime}$ & $15^{\prime}$ & $30^{\prime}$ & $60^{\prime}$ & $120^{\prime}$ & $180^{\prime}$ \\
\hline Lagarta & $0,96 \mathrm{a}$ & $1,04 \mathrm{a}$ & $1,09 \mathrm{a}$ & $1,09 \mathrm{a}$ & $1,10 \mathrm{a}$ & $1,07 \mathrm{a}$ & $1,02 \mathrm{a}$ & $1,05 \mathrm{a}$ & $1,11 \mathrm{a}$ \\
\hline Lagarta + Folha & $0,71 \mathrm{a}$ & $0,79 \mathrm{a}$ & $0,79 \mathrm{a}$ & $0,85 \mathrm{a}$ & $0,90 \mathrm{a}$ & $0,90 \mathrm{a}$ & $1,03 \mathrm{a}$ & $1,19 \mathrm{a}$ & $1,24 \mathrm{a}$ \\
\hline Lagarta + Fezes & $1,02 \mathrm{a}$ & $1,03 \mathrm{a}$ & $0,98 \mathrm{a}$ & $1,00 \mathrm{a}$ & $1,04 \mathrm{a}$ & $1,12 \mathrm{a}$ & $1,08 \mathrm{a}$ & $1,32 \mathrm{a}$ & $1,33 \mathrm{a}$ \\
\hline Lagarta + Folha + Fezes & $0,71 \mathrm{a}$ & $0,76 \mathrm{a}$ & $0,74 \mathrm{a}$ & $0,76 \mathrm{a}$ & $0,81 \mathrm{a}$ & $0,98 \mathrm{a}$ & $1,01 \mathrm{a}$ & $1,07 \mathrm{a}$ & $1,21 \mathrm{a}$ \\
\hline F (Tratamento) & $3,59^{\mathrm{NS}}$ & $1,65^{\mathrm{NS}}$ & $1,84^{\mathrm{NS}}$ & $1,78^{\mathrm{NS}}$ & $0,97^{\mathrm{NS}}$ & $0,45^{\mathrm{NS}}$ & $0,04^{\mathrm{NS}}$ & $0,81^{\mathrm{NS}}$ & $0,41^{\mathrm{NS}}$ \\
\hline \multirow[t]{3}{*}{ C.V. $(\%)$} & 32,43 & 40,68 & 40,34 & 38,4 & 44,37 & 44,96 & 45,04 & 38,35 & 37,5 \\
\hline & \multicolumn{9}{|c|}{ Ninfas de $P$. nigrispinus predando lagartas de $S$. cosmioides } \\
\hline & \multicolumn{9}{|c|}{ TEMPOS $^{1}$} \\
\hline TRATAMENTOS & $1^{\prime}$ & $3^{\prime}$ & $5^{\prime}$ & $10^{\prime}$ & $15^{\prime}$ & $30^{\prime}$ & $60^{\prime}$ & $120^{\prime}$ & $180^{\prime}$ \\
\hline Lagarta & $0,00 \mathrm{a}$ & $0,00 \mathrm{a}$ & $0,10 \mathrm{a}$ & $0,00 \mathrm{a}$ & $0,00 \mathrm{a}$ & $0,20 \mathrm{a}$ & $0,10 \mathrm{a}$ & $0,20 \mathrm{a}$ & $0,30 \mathrm{a}$ \\
\hline Lagarta + Folha & $0,00 \mathrm{a}$ & $0,00 \mathrm{a}$ & $0,00 \mathrm{a}$ & $0,00 \mathrm{a}$ & $0,00 \mathrm{a}$ & $0,00 \mathrm{a}$ & $0,50 \mathrm{a}$ & $0,80 \mathrm{a}$ & $1,00 \mathrm{a}$ \\
\hline Lagarta + Fezes & $0,00 \mathrm{a}$ & $0,00 \mathrm{a}$ & $0,10 \mathrm{a}$ & $0,30 \mathrm{a}$ & $0,20 \mathrm{a}$ & $0,30 \mathrm{a}$ & $0,60 \mathrm{a}$ & $1,00 \mathrm{a}$ & $1,10 \mathrm{a}$ \\
\hline Lagarta + Folha + Fezes & $0,00 \mathrm{a}$ & $0,00 \mathrm{a}$ & $0,00 \mathrm{a}$ & $0,00 \mathrm{a}$ & $0,00 \mathrm{a}$ & $0,10 \mathrm{a}$ & $0,50 \mathrm{a}$ & $0,70 \mathrm{a}$ & $1,00 \mathrm{a}$ \\
\hline F (Tratamento) & $9,00^{\mathrm{NS}}$ & $9,00^{\mathrm{NS}}$ & $0,64^{\mathrm{NS}}$ & $2,08^{\mathrm{NS}}$ & $1,00^{\mathrm{NS}}$ & $0,46^{\mathrm{NS}}$ & $0,68^{\mathrm{NS}}$ & $1,14^{\mathrm{NS}}$ & $1,08^{\mathrm{NS}}$ \\
\hline C.V. $(\%)$ & 6,20 & 6,20 & 16,08 & 20,57 & 18,96 & 30,45 & 39,63 & 39,95 & 40,22 \\
\hline
\end{tabular}

${ }^{1}$ Médias seguidas de mesma letra, na coluna, não diferem significativamente entre si pelo teste de Tukey, a 5\% de probabilidade. Para análise, os dados foram transformados em $(x+0,5)^{1 / 2}$

Tabela 2. Número médio de adultos de Podisus nigrispinus atraídos e predando lagartas de Spodoptera cosmioides nos diferentes tratamentos e tempos de avaliação. Temperatura: $25 \pm 1{ }^{\circ}$ C; U.R.: $70 \pm 10 \%$; Fotofase: 12 horas.

\begin{tabular}{|c|c|c|c|c|c|c|c|c|c|}
\hline \multirow{3}{*}{ TRATAMENTOS } & \multicolumn{9}{|c|}{ Adultos de $P$. nigrispinus atraídos por lagartas de $S$. cosmioides } \\
\hline & \multicolumn{9}{|c|}{ TEMPOS $^{1}$} \\
\hline & $1^{\prime}$ & $3^{\prime}$ & $5^{\prime}$ & $10^{\prime}$ & $15^{\prime}$ & $30^{\prime}$ & $60^{\prime}$ & $120^{\prime}$ & $180^{\prime}$ \\
\hline Lagarta & $0,81 \mathrm{a}$ & $0,91 \mathrm{a}$ & $0,86 \mathrm{a}$ & $0,97 \mathrm{a}$ & $0,96 \mathrm{a}$ & $0,91 \mathrm{a}$ & $0,97 \mathrm{a}$ & $0,86 \mathrm{a}$ & $0,86 \mathrm{a}$ \\
\hline Lagarta+Folha & $0,71 \mathrm{a}$ & $0,81 \mathrm{a}$ & $0,91 \mathrm{a}$ & $0,76 \mathrm{a}$ & $0,76 \mathrm{a}$ & $0,90 \mathrm{a}$ & $1,05 \mathrm{a}$ & $1,14 \mathrm{a}$ & $1,11 \mathrm{a}$ \\
\hline Lagarta+Fezes & $0,90 \mathrm{a}$ & $0,76 \mathrm{a}$ & $0,76 \mathrm{a}$ & $0,81 \mathrm{a}$ & $0,86 \mathrm{a}$ & $0,81 \mathrm{a}$ & $0,85 \mathrm{a}$ & $0,90 \mathrm{a}$ & $1,00 \mathrm{a}$ \\
\hline Lagarta+Folha+Fezes & $0,91 \mathrm{a}$ & $0,86 \mathrm{a}$ & $0,86 \mathrm{a}$ & $0,91 \mathrm{a}$ & $0,91 \mathrm{a}$ & $1,00 \mathrm{a}$ & $1,00 \mathrm{a}$ & $1,04 \mathrm{a}$ & $1,14 \mathrm{a}$ \\
\hline $\mathrm{F}$ (Tratamento) & $1,78^{\mathrm{NS}}$ & $1,00^{\mathrm{NS}}$ & $0,81^{\mathrm{NS}}$ & $1,80^{\mathrm{NS}}$ & $1,34^{\mathrm{NS}}$ & $0,63^{\mathrm{NS}}$ & $0,72^{\mathrm{NS}}$ & $1,55^{\mathrm{NS}}$ & $1,45^{\mathrm{NS}}$ \\
\hline \multirow[t]{3}{*}{ C.V. $(\%)$} & 27,09 & 25,26 & 26,93 & 25,83 & 27,58 & 34,32 & 33,81 & 33,12 & 31,94 \\
\hline & \multicolumn{9}{|c|}{ Adultos de $P$. nigrispinus predando lagartas de $S$. cosmioides } \\
\hline & \multicolumn{9}{|c|}{ TEMPOS $^{1}$} \\
\hline TRATAMENTOS & $1^{\prime}$ & $3^{\prime}$ & $5^{\prime}$ & $10^{\prime}$ & $15^{\prime}$ & $30^{\prime}$ & $60^{\prime}$ & $120^{\prime}$ & $180^{\prime}$ \\
\hline Lagarta & $0,10 \mathrm{a}$ & $0,10 \mathrm{a}$ & $0,10 \mathrm{a}$ & $0,20 \mathrm{a}$ & $0,20 \mathrm{a}$ & $0,10 \mathrm{a}$ & $0,40 \mathrm{a}$ & $0,20 \mathrm{a}$ & $0,20 \mathrm{a}$ \\
\hline Lagarta+Folha & $0,00 \mathrm{a}$ & $0,00 \mathrm{a}$ & $0,00 \mathrm{a}$ & $0,00 \mathrm{a}$ & $0,10 \mathrm{a}$ & $0,10 \mathrm{a}$ & $0,40 \mathrm{a}$ & $0,20 \mathrm{a}$ & $0,60 \mathrm{a}$ \\
\hline Lagarta+Fezes & $0,00 \mathrm{a}$ & $0,00 \mathrm{a}$ & $0,00 \mathrm{a}$ & $0,00 \mathrm{a}$ & $0,00 \mathrm{a}$ & $0,00 \mathrm{a}$ & $0,30 \mathrm{a}$ & $0,20 \mathrm{a}$ & $0,60 \mathrm{a}$ \\
\hline Lagarta+Folha+Fezes & $0,10 \mathrm{a}$ & $0,10 \mathrm{a}$ & $0,10 \mathrm{a}$ & $0,10 \mathrm{a}$ & $0,10 \mathrm{a}$ & $0,30 \mathrm{a}$ & $0,40 \mathrm{a}$ & $0,60 \mathrm{a}$ & $0,70 \mathrm{a}$ \\
\hline $\mathrm{F}$ (Tratamento) & $0,64^{\mathrm{NS}}$ & $0,64^{\mathrm{NS}}$ & $0,64^{\mathrm{NS}}$ & $1,25^{\mathrm{NS}}$ & $0,78^{\mathrm{NS}}$ & $0,84^{\mathrm{NS}}$ & $0,09^{\mathrm{NS}}$ & $1,13^{\mathrm{NS}}$ & $1,10^{\mathrm{NS}}$ \\
\hline C.V. $(\%)$ & 16,08 & 16,08 & 16,08 & 18,77 & 19,91 & 26,00 & 35,49 & 31,68 & 32,16 \\
\hline
\end{tabular}

${ }^{1}$ Médias seguidas de mesma letra, na coluna, não diferem significativamente entre si pelo teste de Tukey, a 5\% de probabilidade. Para análise, os dados foram transformados em $(x+0,5)^{1 / 2}$ 
De modo geral, observa-se que embora as ninfas e os adultos tenham sido atraídos de forma relativamente rápida, isso não se traduz em predação, presumindo-se que esse predador necessita de um tempo maior para a realização dessa atividade (Tabelas 1 e 2). O fato de $P$. nigrispinus estar se alimentando anteriormente ao teste com larvas de $T$. molitor e de que, em geral, predadores apresentam grande capacidade de armazenamento de energia podem explicar o maior tempo necessário para o início de atividade de predação.

Além disso, o semelhante comportamento de atratividade do predador aos diferentes tratamentos pode ser explicado pelo fato de as lagartas de $S$. cosmioides não estarem se alimentando e consequentemente provocando injúrias nas folhas de soja durante $o$ experimento. Os compostos induzidos pela fitofagia são diferentes daqueles emitidos por plantas que não foram injuriadas por insetos fitófagos (DICKE, 1999), podendo ter efeito repelente sobre estes ou atraente aos parasitoides e predadores (PRICE, 1997; CHEN, 2008).

Segundo Pfannestiel et al. (1995), percevejos predadores, em geral, localizam suas presas, principalmente por meio de fezes, por sinais visuais a pequenas distâncias. Fato corroborado por Cavalcanti et al. (2000), cujos estudos mostraram que o predador $P$. nigrispinus pode localizar lagartas de $T$. arnobia combinando sinomônio da planta hospedeira e cairomônios das fezes e do corpo da presa.

Observou-se que, com exceção do tratamento constituído apenas por lagartas, a regressão entre o número de ninfas ou adultos atraídos em função do tempo em relação aos demais tratamentos, quais sejam, lagarta + folha, lagarta + fezes e lagarta + folha + fezes, apresentou diferença significativa (Figura 1). A curva do número de adultos de $P$. nigrispinus atraídos ao tratamento lagarta + folha em função do tempo se ajustou à regressão polinomial de $2^{\circ}$ grau, enquanto as regressões entre o número de ninfas atraídas ao tratamento lagarta + folha e de ninfas ou adultos em relação à lagarta + fezes e lagarta + folha + fezes se ajustaram ao modelo linear, de maneira que o número de percevejos aumentou significativamente ao longo do tempo.

Esses resultados ajudam a demonstrar a ação dos aleloquímicos liberados pelas folhas de soja e pelas fezes de $S$. cosmioides traduzindo em maior atratividade de $P$. nigrispinus em comparação à resposta apenas dos voláteis emitidos pela presa.

Com base nos resultados obtidos, pelo fato de numericamente haver maior número de $P$. nigrispinus sobre os tratamentos constituídos de mais outro substrato, além de apenas lagarta, passível de também estar liberando voláteis atraentes ao percevejo, convencionou-se a utilização de lagarta + folha da cultivar a ser testada + fezes da lagarta alimentada com a mesma cultivar visando à avaliação do efeito de cultivares resistentes e suscetíveis de soja no comportamento de forrageamento do predador.

No teste de atratividade de ninfas de $P$. nigrispinus por lagartas de $S$. cosmioides alimentadas em cultivares de soja com diferentes graus de resistência a insetos, constatou-se, como no teste anterior, que não houve diferença significativa do número médio de ninfas ou adultos do predador nos diferentes tratamentos (Tabelas 3 e 4). No entanto, lagartas alimentadas com os genótipos IGRA RA 626 RR e IAC 100 apresentaram tendência de serem mais atrativas para as ninfas do predador nos primeiros minutos de avaliação, com respectivas médias de 0,70 e 0,50 indivíduos após um minuto da liberação dos insetos. Nos demais tratamentos, nesse tempo, nenhuma ninfa tinha sido atraída para lagartas alimentadas com os genótipos Dowling e BR 16 (Tabela 3). 
Comportamento de Podisus nigrispinus (Dallas) (Hemiptera: Pentatomidae) na interação com lagartas de Spodoptera cosmioides (Walker) (Lepidoptera: Noctuidae) e cultivares de soja com diferentes graus de resistência

Lagarta

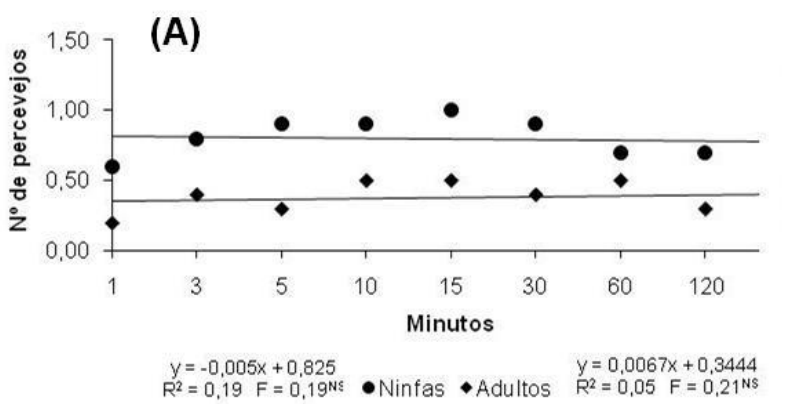

Lagarta + Fezes

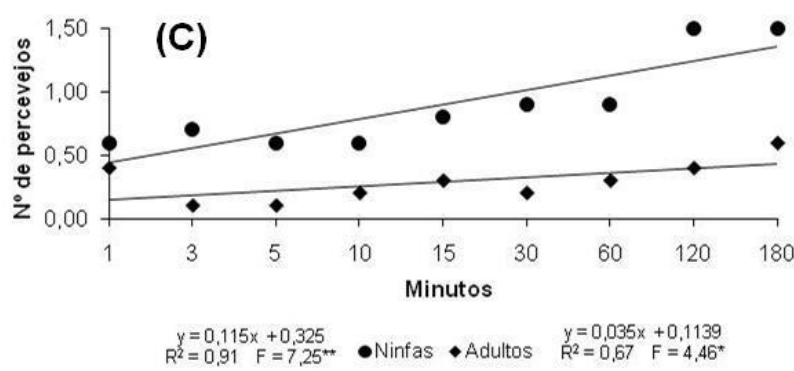

Lagarta + Folha
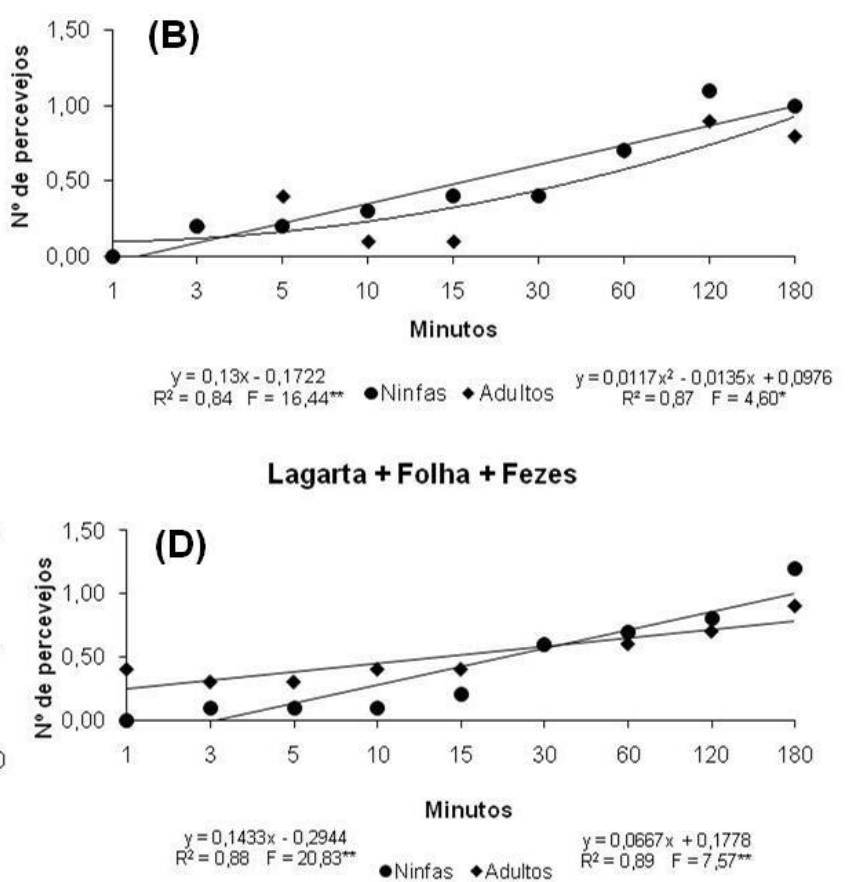

Figura 1. Regressão entre o número médio de ninfas e adultos de Podisus nigrispinus atraídos por lagartas de Spodoptera cosmioides na presença ou ausência de folhas de soja (cultivar BR 16) e fezes em função do tempo.

No tempo de 180 minutos, todos os tratamentos mostraram tendência, em relação aos tempos precedentes, de serem atrativos para ninfas do predador, com médias de 0,40 ; 0,$80 ; 1,00$ e 1,10 para lagartas de $S$. cosmioides alimentadas com os genótipos IGRA RA 626 RR, BR 16, Dowling e IAC 100 , respectivamente (Tabela 3 ).

Quanto aos indivíduos realizando predação, observou-se número bastante reduzido nos primeiros minutos. Somente nas últimas avaliações verificou-se maior número de ninfas de $P$. nigrispinus predando lagartas, com médias de 0,20;0,50;0,60 e 0,70 para aquelas alimentadas com as cultivares IGRA RA 626 RR, BR 16, IAC 100 e Dowling, respectivamente, no tempo de 180 minutos (Tabela 3).

Pode-se observar que lagartas alimentadas com o genótipo Dowling foram, numericamente, menos atrativas aos adultos do percevejo durante toda a avaliação, com número médio de $P$. nigrispinus variando de 0,10 a 0,30 , enquanto os tratamentos com lagartas alimentadas com os genótipos BR 16, IGRA RA 626 RR e IAC 100 apresentaram tendência de maior número de predadores atraídos, com médias de 0,50 a 0,60 indivíduos por tratamento aos 180 minutos (Tabela 4).

Os tratamentos BR 16 e IAC 100, de modo geral, apresentaram comportamento semelhante para atração e predação de adultos de $P$. nigrispinus, mostrando, numericamente, maiores médias para esses parâmetros em comparação aos tratamentos IGRA RA 626 RR e Dowling (Tabela 4).

Jesus (2009), avaliando a atratividade do predador $P$. nigrispinus por lagartas de Spodoptera frugiperda (J. E. Smith, 1797) (Lepidoptera: Noctuidae), constatou que as lagartas alimentadas na cultivar de algodoeiro Delta Opal foram as mais atrativas, diferindo 
significativamente daquelas criadas nas cultivares FMX 910 e FMX 966.

Bartlett (2008), estudando a interação entre genótipos resistente e suscetível de soja, o herbívoro Epilachna varivestis Mulsant, 1850 (Coleoptera: Coccinellidae) e o predador Podisus maculiventris (Say, 1832) (Hemiptera: Pentatomidae) verificou que o inimigo natural preferiu se alimentar do besouro mexicano que se alimentou do genótipo suscetível Cobb em comparação ao resistente PI 229358, além de o primeiro genótipo em associação com o agente de controle biológico ter sido significativamente mais produtivo.

Matos Neto et al. (2002) estudaram o efeito da alimentação de $P$. nigrispinus com folhas das cultivares de soja resistente IAC 17 e suscetível UFV 16 e lagartas de Anticarsia gemmatalis Hübner, 1818 (Lepidoptera: Noctuidae) alimentadas com essas cultivares e concluíram que IAC 17 exerceu um efeito deletério sobre o predador, reduzindo significativamente o número de fêmeas que ovipositaram e numericamente o número de ovos por fêmea, postura por fêmea, ninfas por fêmea e presas mortas por fêmea em comparação com a cultivar suscetível.

Cordeiro (2003), estudando o desenvolvimento de $P$. nigrispinus alimentados com pupas de T. molitor e as cultivares de soja resistente IAC 100 e suscetível UFV 16, constatou efeito deletério da cultivar resistente na fase ninfal apenas para a duração do quinto ínstar e da fase completa, porém, a viabilidade e peso de ninfas não foi afetada. No estágio adulto, a única característica que foi afetada por IAC 100 foi o número de ovos por fêmea, entretanto, a viabilidade de ovos foi superior nessa cultivar. Contudo, a maioria das características biológicas e da tabela de vida de $P$. nigrispinus encontradas nesse trabalho foram semelhantes entre as cultivares resistentes e suscetíveis de soja.
Levando-se em consideração a integração dos métodos de resistência de plantas e controle biológico, a observação de ausência de qualquer influência adversa das cultivares resistentes IAC 100 e Dowling na atratividade e predação de $P$. nigrispinus a lagartas de $S$. cosmioides alimentadas com essas cultivares pode tornar essa associação ainda mais eficiente no controle da praga em campo, podendo ocorrer tanto um efeito independente de cada uma das táticas como um efeito sinérgico de ambas.

Ao se avaliar o comportamento de busca de ninfas ou adultos de $P$. nigrispinus às lagartas de $S$. cosmioides alimentadas em cada cultivar de soja, pode-se verificar que, de modo geral, houve diferença significativa do número de percevejos atraídos em função do tempo, com exceção dos números de ninfas do predador em relação às lagartas criadas nas cultivares IGRA RA 626 RR e IAC 100 e de adultos em Dowling, que foram praticamente constantes durante todo o experimento (Figura 2).

Todavia, o número de ninfas ou adultos de $P$. nigrispinus atraídos às lagartas alimentadas com a cultivar BR 16, número de ninfas atraídas às lagartas criadas com Dowling e de adultos dos percevejos em relação a $S$. cosmioides alimentados com IGRA RA 626 RR e IAC 100 aumentaram significativamente em função do tempo, sendo que a regressão entre os fatores número de predadores e tempo se ajustou ao modelo linear (Figura 2).

O mesmo comportamento pode ser observado no trabalho realizado por Angelini \& Boiça Júnior (2009), avaliando a atratividade de $P$. nigrispinus por lagartas de Dione juno juno (Cramer, 1779) (Lepidoptera: Nymphalidae) criadas em diferentes genótipos de maracujazeiro, onde a atratividade aumentou após uma hora do estabelecimento do experimento. 
Comportamento de Podisus nigrispinus (Dallas) (Hemiptera: Pentatomidae) na interação com lagartas de Spodoptera cosmioides (Walker) (Lepidoptera: Noctuidae) e cultivares de soja com diferentes graus de resistência

Tabela 3. Número médio de ninfas de $3^{\circ}$ ínstar de Podisus nigrispinus, atraídos e predando lagartas de Spodoptera cosmioides nos diferentes genótipos de soja e tempos de avaliação. Temperatura: $25 \pm 1{ }^{\circ} \mathrm{C}$; U.R.: $70 \pm 10 \%$; Fotofase: 12 horas.

\begin{tabular}{|c|c|c|c|c|c|c|c|c|c|}
\hline \multirow{3}{*}{ CULTIVARES } & \multicolumn{9}{|c|}{ Ninfas de $P$. nigrispinus atraídos por lagartas de $S$. cosmioides } \\
\hline & \multicolumn{9}{|c|}{ TEMPOS $^{1}$} \\
\hline & $1^{\prime}$ & $3^{\prime}$ & $5^{\prime}$ & $10^{\prime}$ & $15^{\prime}$ & $30^{\prime}$ & $60^{\prime}$ & $120^{\prime}$ & $180^{\prime}$ \\
\hline BR 16 & $0,00 \mathrm{a}$ & $0,30 \mathrm{a}$ & $0,20 \mathrm{a}$ & $0,50 \mathrm{a}$ & $0,50 \mathrm{a}$ & $0,50 \mathrm{a}$ & $0,90 \mathrm{a}$ & $1,00 \mathrm{a}$ & $\overline{0,80 \mathrm{a}}$ \\
\hline Dowling & $0,00 \mathrm{a}$ & $0,00 \mathrm{a}$ & $0,20 \mathrm{a}$ & $0,20 \mathrm{a}$ & $0,30 \mathrm{a}$ & $0,40 \mathrm{a}$ & $0,50 \mathrm{a}$ & $0,90 \mathrm{a}$ & $1,00 \mathrm{a}$ \\
\hline IGRA RA 626 RR & $0,70 \mathrm{a}$ & $0,60 \mathrm{a}$ & $0,50 \mathrm{a}$ & $0,60 \mathrm{a}$ & $0,50 \mathrm{a}$ & $0,80 \mathrm{a}$ & $0,50 \mathrm{a}$ & $0,60 \mathrm{a}$ & $0,40 \mathrm{a}$ \\
\hline IAC 100 & $0,50 \mathrm{a}$ & $0,50 \mathrm{a}$ & $0,60 \mathrm{a}$ & $0,70 \mathrm{a}$ & $0,80 \mathrm{a}$ & $0,70 \mathrm{a}$ & $0,80 \mathrm{a}$ & $0,80 \mathrm{a}$ & $1,10 \mathrm{a}$ \\
\hline $\mathrm{F}$ (Tratamento) & $4,00^{\mathrm{NS}}$ & $2,54^{\mathrm{NS}}$ & $1,02^{\mathrm{NS}}$ & $0,73^{\mathrm{NS}}$ & $0,53^{\mathrm{NS}}$ & $0,56^{\mathrm{NS}}$ & $0,52^{\mathrm{NS}}$ & $0,24^{\mathrm{NS}}$ & $1,15^{\mathrm{NS}}$ \\
\hline \multirow[t]{3}{*}{ C.V. $(\%)$} & 31,46 & 30,07 & 33,47 & 39,20 & 40,30 & 40,34 & 35,59 & 40,23 & 37,3 \\
\hline & \multicolumn{9}{|c|}{ Ninfas de $P$. nigrispinus predando lagartas de $S$. cosmioides } \\
\hline & \multicolumn{9}{|c|}{ TEMPOS $^{1}$} \\
\hline CULTIVARES & $1^{\prime}$ & $3 '$ & $5^{\prime}$ & $10^{\prime}$ & $15^{\prime}$ & $30^{\prime}$ & $60^{\prime}$ & $120^{\prime}$ & $180^{\prime}$ \\
\hline BR 16 & $0,00 \mathrm{a}$ & $0,00 \mathrm{a}$ & $0,00 \mathrm{a}$ & $0,20 \mathrm{a}$ & $0,20 \mathrm{a}$ & $0,30 \mathrm{a}$ & $0,50 \mathrm{a}$ & $0,80 \mathrm{a}$ & $0,50 \mathrm{a}$ \\
\hline Dowling & $0,00 \mathrm{a}$ & $0,00 \mathrm{a}$ & $0,00 \mathrm{a}$ & $0,10 \mathrm{a}$ & $0,10 \mathrm{a}$ & $0,30 \mathrm{a}$ & $0,30 \mathrm{a}$ & $0,50 \mathrm{a}$ & $0,70 \mathrm{a}$ \\
\hline IGRA RA 626 RR & $0,20 \mathrm{a}$ & $0,20 \mathrm{a}$ & $0,10 \mathrm{a}$ & $0,10 \mathrm{a}$ & $0,10 \mathrm{a}$ & $0,20 \mathrm{a}$ & $0,20 \mathrm{a}$ & $0,20 \mathrm{a}$ & $0,20 \mathrm{a}$ \\
\hline IAC 100 & $0,10 \mathrm{a}$ & $0,10 \mathrm{a}$ & $0,10 \mathrm{a}$ & $0,00 \mathrm{a}$ & $0,00 \mathrm{a}$ & $0,00 \mathrm{a}$ & $0,10 \mathrm{a}$ & $0,50 \mathrm{a}$ & $0,60 \mathrm{a}$ \\
\hline F (Tratamento) & $1,25^{\mathrm{NS}}$ & $2,25^{\mathrm{NS}}$ & $0,64^{\mathrm{NS}}$ & $0,71^{\mathrm{NS}}$ & $0,37^{\mathrm{NS}}$ & $0,49^{\mathrm{NS}}$ & $0,52^{\mathrm{NS}}$ & $0,70^{\mathrm{NS}}$ & $0,67^{\mathrm{NS}}$ \\
\hline C.V. $(\%)$ & 18,77 & 14,89 & 16,08 & 24,06 & 24,67 & 34,81 & 34,39 & 39,59 & 39,96 \\
\hline
\end{tabular}

${ }^{1}$ Médias seguidas de mesma letra, na coluna, não diferem significativamente entre si pelo teste de Tukey, a $5 \%$ de probabilidade. Para análise, os dados foram transformados em $(x+0,5)^{1 / 2}$
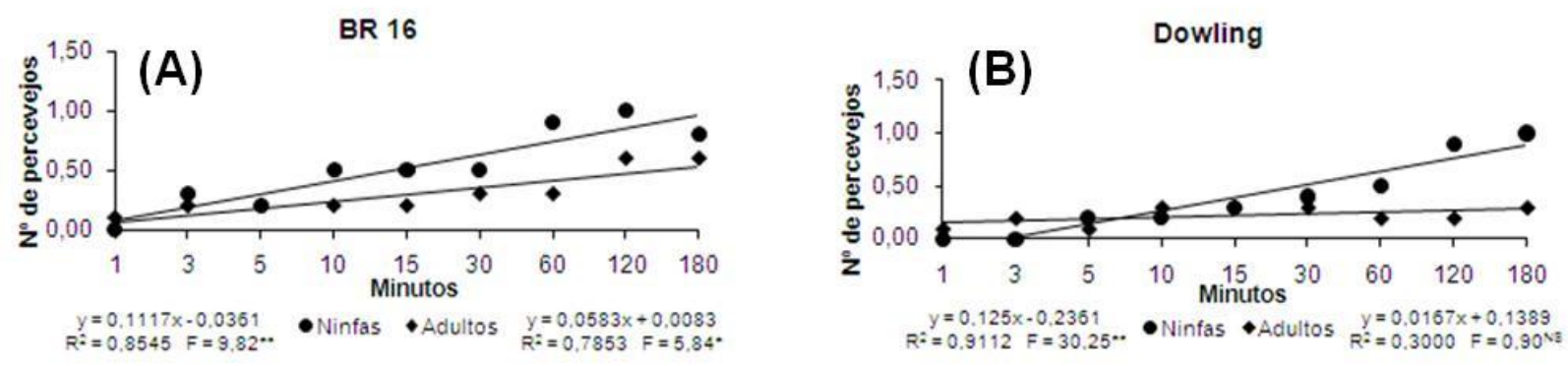

IGRA 626

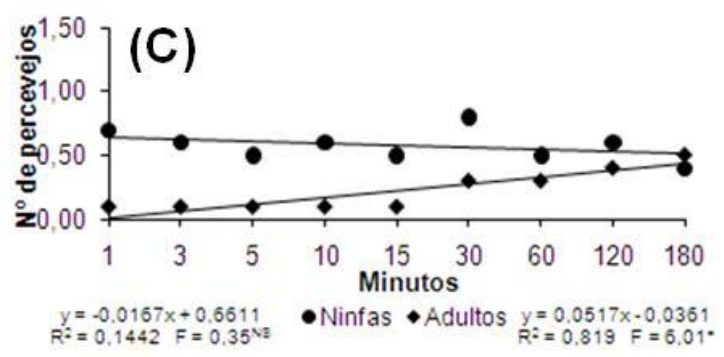

IAC 100

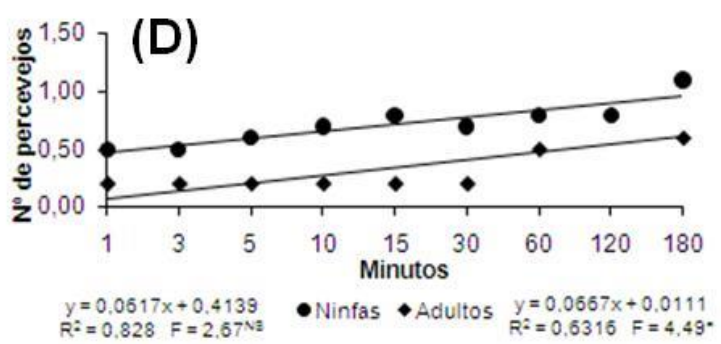

Figura 2. Regressão entre o número médio de ninfas e adultos de Podisus nigrispinus atraídos por lagartas de Spodoptera cosmioides alimentadas com diferentes cultivares de soja em função do tempo. 
Já no trabalho de Jesus (2009), observou-se que a atração de adultos de $P$. nigrispinus por lagartas de $S$. frugiperda, criadas nas cultivares de algodoeiro, ocorreu após duas horas do início do ensaio. Esses resultados sugerem que os tempos de avaliação devem ser maiores para que haja melhor resposta do predador aos odores emitidos.

\section{CONCLUSÕES}

Os voláteis liberados pelas lagartas na presença ou ausência de folhas e fezes mostram-se estimulantes para o comportamento de busca do predador;

As cultivares de soja resistentes IAC 100 e Dowling, utilizadas para a alimentação de lagartas de S. cosmioides, não afetam a atratividade e a capacidade de predação de $P$. nigrispinus;

Para uma resposta mais precisa do predador aos estímulos liberados, recomendase maior tempo de avaliação.

A associação entre cultivares resistentes de soja e controle biológico com $P$. nigripinus pode apresentar um efeito sinérgico no controle de $S$. cosmioides em campo, com potencial para ser incluído em um programa de MIP para a cultura da soja.

\section{REFERÊNCIAS BIBLIOGRÁFICAS}

ANGELINI, M. R.; BOIÇA JÚNIOR, A. L. 2009. Capacidade predatória e atratividade de Podisus nigrispinus (Dallas, 1851) (Hemiptera: Pentatomidae) por lagartas de Dione juno juno (Cramer, 1779) (Lepidoptera: Nymphalidae) criadas em folhas de genótipos de maracujazeiros. Ceres, Viçosa, v. 56, n. 1, p. 25-30.

BARTLETT, R. 2002, Negative interactions between chemical resistance and predators affect fitness in soybeans. Ecological Entomology, London, v. 33, n. 5 , p. 673-678.
BOIÇA JÚNIOR, A. L.; SANTOS, T. M.; SOARES, J. J. 2002. Influência de genótipos de algodoeiro sobre o desenvolvimento e capacidade predatória de ninfas de Podisus nigrispinus (Dallas, 1851). Arquivos do Instituto Biológico, São Paulo, v. 69, n. 1, p. 75-80.

CAVALCANTI, M. G.; VILELA, E. F.; EIRAS, A. E.; ZANUNCIO, J. C.; PICANÇO, M. C. 2000. Interação tritrófica entre Podisus nigrispinus (Dallas) Heteroptera: Pentatomidae), Eucalyptus e lagartas de Thyrinteina arnobia (Stoll) (Lepidoptera: Geometridae): I visitação. Anais da Sociedade Entomológica do Brasil, Jaboticabal, v. 29, n. 4, p. 697-703.

CHEN, M. S. 2008. Inducible direct plant defense against insect herbivores: a review. Insect Science, Beijing, v. 15, n. 2, p. 101-114.

CHIOZZA， M. V.; O' NEAL， M. E.; MACINTOSH, G. C. 2010. Constitutive and induced differential accumulation amino acid in leaves of susceptible and resistant soybean plants in response to the soybean aphid (Hemiptera: Aphididae). Environmental Entomology, College Park, v. 39, n. 3, p. 856-864.

CORDEIRO, J. C. 2003. Efeito do cultivar de soja resistente a insetos IAC 100 no desenvolvimento do predador Podisus nigrispinus (Heteroptera: Pentatomidae) no campo. $65 \mathrm{f}$. Tese (Doutorado em Fitotecnia) Universidade Federal de Viçosa, Viçosa.

DE CLERCQ, P. 2000. Predaceous stinkbugs (Pentatomidae: Asopinae). In: SCHAEFER, C. W.; PANIZZI, A. R. (Ed). Heteroptera of economic importance. Boca Raton: CRC, p. 737789.

DICKE, M. 1999. Are herbivore-induced plant volatiles reliable indicators of herbivore identity to foraging carnivorous arthropods? 
Comportamento de Podisus nigrispinus (Dallas) (Hemiptera: Pentatomidae) na interação com lagartas de Spodoptera cosmioides (Walker) (Lepidoptera: Noctuidae) e cultivares de soja com diferentes graus de resistência

Experimentalis et Applicata, Dordrecht, v. 91, n. 1, p. 131-142.

GREENE, G. L.; LEPPLA, N. C.; DICKERSON, W. A. 1976. Velvet bean caterpillar: a rearing procedure and artificial medium. Journal of Economic Entomology, Lanham, v. 69, n. 4, p. 487488.

HABIB, M. E. M.; PALEARI, M. L.; AMARAL, M. C. E. 1983. Effect of three larval diets on the development of the armyworm, Spodoptera latifascia Walker, 1856 (Noctuidae: Lepidoptera). Revista Brasileira de Zoologia, São Paulo, v. 1, n. 3, p. 177-182.

HILL, C. B.; LI, Y; HARTMAN, G. L. 2004. Resistance to the soybean aphid in soybean germplasm. Crop Science, Madison, v. 44, n. 1, p. 98-106.

ISENHOUR, D. J.; WISEMAN, B. R.; LAYTON, R. C. 1989. Enhanced predation by Orius insiosus (Hymenoptera: Anthocoridae) on larvae of Heliothis zea and Spodoptera frugiperda (Lepidoptera: Noctuidae) caused by prey feeding on resistant corn genotypes. Environmental Entomology, College Park, v. 18, n. 3, p. 418-422.

JESUS, F. G. 2009. Resistência de cultivares de algodoeiro sobre Spodoptera frugiperda e Alabama argillacea (Lepidoptera: Noctuidae) e efeito na biologia e comportamento de Podisus nigrispinus (Hemiptera: Pentatomidae). 85p.Tese (Doutorado em Agronomia - Área de Concentração: Entomologia Agrícola) - Faculdade de Ciências Agrárias e Veterinárias, Universidade Estadual Paulista, Jaboticabal.

KAROWE, D. N.; SCHOONHOVEN, L. M. 1992. Interaction among three trophic levels: the influence of host plant on performance of Pieris brassicae and its parasitoid, Cotesia glomerata. Entomologia Experimentalis et
Applicata, Dordrecht, v. 62, n. 3, p. 241251.

LI, Y., HILL, C. B.; HARTMAN, G. L. 2004. Effect of three resistant soybean genotypes on the fecundity, mortality and maturation of soybean aphid (Homoptera: Aphididae). Journal of Economic Entomology, Lanhan, v. 97, n. 3, p. 11061111.

LOURENÇÃO, A. L.; PEREIRA, J. C. V. N. A.; MIRANDA, M. A. C.; AMBROSANO, G. M. B. 2000. Avaliação de danos causados por percevejos e por lagartas em genótipos de soja de ciclos precoce e semiprecoce. Pesquisa Agropecuária Brasileira, Brasília, v. 35, n. 5, p. 879-886.

MATOS NETO, F. C.; ZANUNCIO, J. C.; PICANÇO, M. C.; CRUZ, I. 2002. Reproductive characteristics of the predator Podisus nigrispinus fed with an insect resistant soybean variety. Pesquisa Agropecuária Brasileira, Brasília, v. 37, n. 7, p. 917-924.

OLIVEIRA, L. J.; HOFFMANN-CAMPO, C. B.; MAZZARIN, R. M. 1993. Aspectos biológicos e nutricionais de Anticarsia gemmatalis Hüb. (Lepidoptera: Noctuidae) em diversos genótipos de soja. Anais da Sociedade Entomológica do Brasil, Jaboticabal, v. 22, n. 33, p. 547-552.

PFANNESTIEL, R. S.; HUNT, R. E.; YEARGAN, K. V. 1995. Orientation of a hemipteran predator to vibrations produced by feeding caterpillars. Journal of Insect Behavior, New York, v. 8, n. 1, p. 1-9.

PIUBELLI, G. C.; HOFFMANN-CAMPO, C. B.; ARRUDA, I. C.; LARA, F. M. 2003. Nymphal development, lipid content, growth and weight gain of Nezara viridula (L.) (Heteroptera: Pentatomidae) fed on soybean genotypes. Neotropical Entomology, Londrina, v. 32 , n. 1, p. 127-132. 
PIUBELLI, G. C.; HOFFMANN-CAMPO, C. B.; MOSCARDI, F.; MIYAKUBO, S. H.; OLIVEIRA, M. C. N. 2005. Are chemical compounds important for soybean resistance to Anticarsia gemmatalis? Journal of Chemical Ecology, New York, v. 31, p. 1509-1525.

PRICE, P. W. 1984. Insect ecology. New York: Wiley-Interscience. 607 p.

RICE, M. E.; WILDE, G. E. 1989. Antibiosis effect of sorghum on the convergent lady beetle (Coleoptera: Coccinellidae), a third-trophic level predator of the greenbug (Homoptera: Aphididae). Journal of Economic Entomology, Lanhan, v. 82, n. 2, p. 570-573.

RUBERSON, J. R.; TAUBER, M. J.; TAUBER, C. A. 1986. Plant feeding by Podisus maculiventris (Hemiptera: Pentatomidae): effect on survival, development and preoviposition period. Environmental Entomology, College Park, v. 15, n. 4, p. 894-897.

SEIGLER, D.; PRICE, P. W. 1976. Secondary compounds in plants: primary functions. American Naturalist, Chicago, v. 110, n. 971, p. 101-105.

SOUZA, B. H. S.; BOIÇA JÚNIOR, A. L.; JANINI, J. C.; SILVA, A. G.; RODRIGUES, N. E. L. 2012. Feeding of Spodoptera eridania (Lepidoptera: Noctuidae) on soybean genotypes. Revista Colombiana de Entomología, Bogotá, v. 38, n. 2, p. 215-223.

SOUZA, B. H. S. 2011. Tipos e graus de resistência de genótipos de soja a Spodoptera eridania (Cramer, 1782) (Lepidoptera: Noctuidae). $73 \mathrm{f}$. Dissertação (Mestrado em Agronomia Área de Concentração: Entomologia Agrícola) - Faculdade de Ciências Agrárias e Veterinárias, Universidade Estadual Paulista, Jaboticabal.

TORRES, J. B.; ZANUNCIO, J. C.; ZANUNCIO, T. V. 1996. Produção e uso de percevejos predadores no controle biológico de pragas florestais. In:
WORKSHOP SOBRE PROTEÇÃO FLORESTAL DO MERCOSUL, 1996, Santa Maria. Anais... Santa Maria: UFSM. p. 41-51.

VET, L. E. M.; DICKE, M. 1992. Ecology of infochemical used by natural enemies in a tritrophic context. Annual Review of Entomology, Stanford, v. 37, p. 141-72.

VIVAN, L. M.; TORRES, J. B.; VEIGA, A. F. S. L. 2003. Development and reproduction of a predatory stinkbug, Podisus nigrispinus in relation to two different prey types and environmental conditions. BioControl, Dordrecht, v. 48, n. 2, p. $155-168$.

ZANUNCIO, J. C.; MOLINA-RUGAMA, A. J.; SERRÃO, J. E.; PRATISSOLI, D. 2001. Nymphal development and reproduction of Podisus nigrispinus (Heteroptera: Pentatomidae) fed with combinations of Tenebrio molitor (Coleoptera: Tenebrionidae) pupae and Musca domestica (Diptera: Muscidae) larvae. Biocontrol Science and Technology, Oxford, v. 11, n. 3, p. 331337.
Recebido em: 22/2/2013 Aceito para publicação em: 01/4/2014 